\title{
Pseudospectral discretization of nonlinear delay equations: new prospects for numerical bifurcation analysis
}

\author{
D. Breda ${ }^{\dagger}$, O. Diekmann ${ }^{\ddagger}$, M. Gyllenberg ${ }^{\S}$, F. Scarabel ${ }^{\S}$, and R. Vermiglio ${ }^{\dagger}$
}

\begin{abstract}
We apply the pseudospectral discretization approach to nonlinear delay models described by delay differential equations, renewal equations or systems of coupled renewal equations and delay differential equations. The aim is to derive ordinary differential equations and to investigate the stability and bifurcation of equilibria of the original model by available software packages for continuation and bifurcation for ordinary differential equations. Theoretical and numerical results confirm the effectiveness and the versatility of the approach, opening a new perspective for the bifurcation analysis of delay equations, in particular coupled renewal and delay differential equations.
\end{abstract}

Key words. Delay differential equations, renewal equations, Volterra delay equations, physiologically structured populations, stability of equilibria, numerical bifurcation, pseudospectral method.

AMS subject classifications. 34K99, 37M20, 65J08, 65L07, 65P30, 92D25.

1. Introduction. In the last decades the interest in differential equations with delay has been increasing, motivated by the fact that the incorporation of the history of the variables in the definition of the evolution law often provides a better description of the real mechanisms. Nowadays the theory of Delay Differential Equations (DDEs), also referred to as retarded functional differential equations, is well established [18, 30]. Models involving delay arise in many different fields $[10,24,32,40]$. In applications to structured population dynamics, Renewal Equations (REs), also known as Volterra functional equations, as well as coupled REs/DDEs, play a central role $[12,13,14,15]$. Relevant examples of the latter are provided by the models of Daphnia type, describing the competition of a size/age structured consumer for an unstructured resource $[17,20]$. In such models the $\mathrm{RE}$ for the population birth rate of the consumer is coupled with the DDE for the resource concentration, with right-hand sides of integral type implicitly defined through external Ordinary Differential Equations (ODEs).

Delay equations are "rules for extending (in one direction) a function that is a priori defined on an interval" [17] and, once the space of the history function is specified, they generate infinite-dimensional dynamical systems. Nevertheless, some of the fundamental results for ODEs have been extended. In this paper the focus is on the stability and bifurcation of equilibria and, in particular, the principle of linearized stability and the related bifurcation

\footnotetext{
${ }^{\dagger}$ Department of Mathematics and Computer Science, University of Udine, via delle Scienze 206, I-33100 Udine, Italy (dimitri.breda@uniud.it, rossana.vermiglio@uniud.it). The work of the authors was supported by the Italian National Scientific Computing Group 2014 grant "Numerical analysis of infinite-dimensional and discontinuous differential problems".

${ }^{\ddagger}$ Department of Mathematics, University of Utrecht, Budapestlaan 6, P.O. Box 80010, 3508 TA Utrecht, The Netherlands (O.Diekmann@uu.nl).

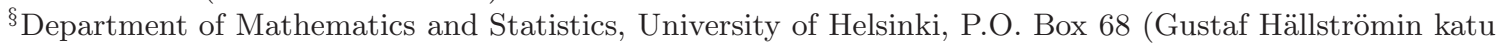
2b), FI-00014 Helsinki, Finland (mats.gyllenberg@helsinki.fi, francesca.scarabel@helsinki.fi). The work of the authors was supported partly by the Finnish Centre of Excellence in Analysis and Dynamics Research (Academy of Finland) and partly by The Finnish Society of Sciences and Letters.
} 
theorems. For DDEs we refer to the books [18, 30], whereas for REs and coupled REs/DDEs a rigorous analysis has been recently developed using sun-star calculus in [17].

For parametrized ODEs there exist efficient numerical methods [34] and software packages for continuation and bifurcation problems, see for instance AUTO, MATCONT, PYDSTOOL $[2,37,39]$ and the dynamical systems software webpage [22] for further references. These toolboxes offer various possibilities, such as continuation of equilibria and cycles, detection and normal form analysis of bifurcations, their continuation in two and three parameters, branch switching and numerical computation of periodic orbits. Due to the high complexity of delay equations, numerical methods are necessary, but also more difficult to develop. Indeed, packages like DDE-BIFTOOL, KNUT, XPP, etc. [11, 22, 33, 43], are either limited in the range of bifurcations they can analyze or in the class of models they can be applied to (e.g., only DDEs, only discrete delays). Therefore, very general toolboxes for DDEs, REs and coupled REs/DDEs are not available, despite the broad and increasing attention for the latter in view of applications to physiologically structured population models. These considerations motivate the present paper.

Different numerical methods have been proposed for the stability analysis of equilibria of DDEs (see, e.g., [9, 32] and the references therein) and coupled REs/DDEs [13]. Here, in particular, we focus on the so-called IG-approach developed for linear DDEs, REs and coupled REs/DDEs $[4,5,6,7,8,9]$. It is based on the pseudospectral method $[29,41]$ to discretize the infinitesimal generator associated with linear(ized) delay equations. The spectrum of the resulting linear finite-dimensional operator approximates part of the eigenvalues of the original one with spectral accuracy [41]. This allows to investigate the stability of the equilibrium.

In this paper we propose a new perspective, which consists in the extension of the pseudospectral discretization to the nonlinear case in order to derive nonlinear ODEs. Through the latter, by means of the available numerical software for ODEs, we approximate the dynamics of the original infinite-dimensional systems and construct bifurcation and stability diagrams varying one or two parameters, respectively. The starting point is the equivalent formulation of the delay equation as a nonlinear Abstract Differential Equation (ADE), whose pseudospectral discretization provides the nonlinear ODE. After showing that the equilibria are in one-to-one correspondence and that pseudospectral discretization and linearization commute, we can exploit the principle of linearized stability and the spectral convergence of the eigenvalues in the linear case to analyze the stability and bifurcation of equilibria, thus achieving the goal. The same program can be applied to different classes of nonlinear delay models, namely defined by DDEs, REs or coupled REs/DDEs. We supplement the theoretical results with several numerical tests, showing the effectiveness and flexibility of the approach.

We remind that the idea of studying the bifurcation properties of nonlinear DDEs with one discrete delay through nonlinear ODEs appears already in [25], where the authors discretize the nonlinear $\mathrm{ADE}$ using piecewise linear interpolation on equidistant nodes.

The paper is organized as follows. In $\S 2$ we present the approach for nonlinear DDEs with all the details and proofs, whereas in $\S 3$ we deal with nonlinear REs, emphasizing the differences w.r.t. DDEs. In $\S 4$ we shortly present the discretization of nonlinear coupled REs/DDEs, obtained by combining the previous techniques. Finally, in $\S 5$, we validate the approach presenting numerical tests on various examples obtained by using MATCONT [37], a MATLAB bifurcation toolbox. 
2. Delay differential equations. In this section we introduce the basic notation and we recall the results strictly necessary to derive the formulation of the ADE, which is the starting point of our approach. For a more detailed treatment of the theory of DDEs and linear semigroups, we refer to $[18,23]$.

For $d \in \mathbb{N}$ and $\tau>0$, we consider the Banach space $Y:=\mathcal{C}\left([-\tau, 0] ; \mathbb{R}^{d}\right)$ equipped with the norm $\|\psi\|_{Y}=\max _{\theta \in[-\tau, 0]}|\psi(\theta)|, \psi \in Y$, where $|\cdot|$ is any vector norm in $\mathbb{R}^{d}$.

A nonlinear autonomous DDE has the form

$$
y^{\prime}(t)=G\left(y_{t}\right), t \geq 0
$$

where $y_{t} \in Y$ represents the state, i.e., the history of $y$, that is

$$
y_{t}(\theta):=y(t+\theta), \theta \in[-\tau, 0],
$$

and $G: Y \rightarrow \mathbb{R}^{d}$ is a smooth nonlinear map. Given $\psi \in Y$, the initial value problem for (2.1), i.e.,

$$
\left\{\begin{array}{l}
y^{\prime}(t)=G\left(y_{t}\right), t \geq 0 \\
y(\theta)=\psi(\theta), \theta \in[-\tau, 0]
\end{array}\right.
$$

has a unique solution, which depends continuously on $\psi$. This makes it possible to define the nonlinear solution operators $T(t): Y \rightarrow Y$

$$
T(t)(\psi)=y_{t}, t \geq 0,
$$

associating to the initial state $\psi$ the state $y_{t}$ at time $t \geq 0$. Clearly these form a semigroup of operators $\{T(t)\}_{t \geq 0}$. Since the action of $T(t)$ is translation with a rule for extension defined through (2.1), the corresponding generator $\mathcal{A}_{G}: \operatorname{dom}\left(\mathcal{A}_{G}\right) \subseteq Y \rightarrow Y$ is differentiation, i.e.,

$$
\mathcal{A}_{G}(\psi)=\lim _{t \rightarrow 0^{+}} \frac{1}{t}(T(t)(\psi)-\psi)=\psi^{\prime}, \psi \in \operatorname{dom}\left(\mathcal{A}_{G}\right)
$$

with

$$
\begin{aligned}
\operatorname{dom}\left(\mathcal{A}_{G}\right) & =\{\psi \in Y \mid \text { the limit in }(2.4) \text { exists }\} \\
& =\left\{\psi \in \mathcal{C}^{1}\left([-\tau, 0], \mathbb{R}^{d}\right) \mid \psi^{\prime}(0)=G(\psi)\right\} .
\end{aligned}
$$

The subscript of $\mathcal{A}_{G}$ stresses the dependence of the generator, via the domain (2.5), on the function $G$.

For a linear DDE

$$
y^{\prime}(t)=L y_{t}, t \geq 0,
$$

where $L: Y \rightarrow \mathbb{R}^{d}$ is a linear and bounded operator, the domain condition in (2.5) reads $\psi^{\prime}(0)=L \psi$ and the resulting operator $\mathcal{A}_{L}$ is linear and it is exactly the infinitesimal generator of the strongly continuous linear solution semigroup defined through (2.3). 
The operator (2.4-2.5) allows to define the ADE on $Y$

$$
\frac{d}{d t} v(t)=\mathcal{A}_{G}(v(t)), t \geq 0,
$$

describing the evolution of the state $v(t)=y_{t}$ in the state space $Y$. In fact, it can be shown that for $\psi \in \operatorname{dom}\left(\mathcal{A}_{G}\right)$ the initial value problem (2.2) is equivalent to the abstract Cauchy problem associated with (2.7), i.e.,

$$
\left\{\begin{array}{l}
\frac{d}{d t} v(t)=\mathcal{A}_{G}(v(t)), t \geq 0 \\
v(0)=\psi
\end{array}\right.
$$

in the sense that $v(t)=y_{t}$ is a (classical) solution of (2.8) and vice-versa. We recall that a (classical) solution of (2.8) is a continuously differentiable function $v$ s.t. $v(t) \in \operatorname{dom}\left(\mathcal{A}_{G}\right)$ for all $t \geq 0$, and (2.8) holds. The important point is that for $\psi \in \operatorname{dom}\left(\mathcal{A}_{G}\right)$, the solution $y$ is continuously differentiable on $[-\tau,+\infty)$. It is possible to extend the equivalence to initial states $\psi \in Y$ by rewriting the differential equation (2.7) as an abstract integral equation and by introducing the concept of mild solution (see [23] for the linear case).

2.1. Pseudospectral discretization. Having in mind (2.7), we propose to discretize the infinite-dimensional operator $\mathcal{A}_{G}(2.4-2.5)$ in order to obtain a nonlinear ODE, whose dynamics can be analyzed by the available bifurcation tools. For the linear DDE (2.6) a numerical approach, called IG-approach, has been developed to approximate the eigenvalues of $\mathcal{A}_{L}$ by turning the original eigenvalue problem into an eigenvalue problem for a finite-dimensional linear operator (see the recent monograph [9]). The discretization of $\mathcal{A}_{L}$ is based on the pseudospectral method, which consists in applying the exact action of $\mathcal{A}_{L}$ and the linear condition characterizing its domain to interpolating polynomials at a selected set of points. The spectral accuracy of the IG-approach allows to obtain very accurate approximations of the eigenvalues and this will be important in the stability analysis of equilibria in $\S 2.2$. Here we put forward the pseudospectral discretization for the nonlinear DDE (2.1).

Let $M \in \mathbb{N}$. Given $M$ distinct points in $[-\tau, 0)$, namely $-\tau \leq \theta_{M, M}<\cdots<\theta_{M, 1}<0$, we define

$$
\Omega_{M}:=\left\{\theta_{M, i}, i=1, \ldots, M\right\}
$$

and, motivated by the domain condition in (2.5), we add $\theta_{M, 0}:=0$ and consider

$$
\left\{\theta_{M, 0}=0\right\} \cup \Omega_{M} .
$$

Here we take the $M+1$ extrema Chebyshev nodes relevant to the delay interval $[-\tau, 0]$, i.e.,

$$
\theta_{M, i}=\frac{\tau}{2}\left(\cos \left(\frac{i \pi}{M}\right)-1\right), i=0,1, \ldots, M
$$

The space $Y_{M}:=\left(\mathbb{R}^{d}\right)^{\{0\} \cup \Omega_{M}} \cong \mathbb{R}^{d} \times \mathbb{R}^{d M}$ represents the discretization of $Y$ of index $M$, in the sense that every $\psi \in Y$ is discretized by the column vector $\left(\mathrm{y}, \Psi_{M}\right) \in Y_{M}$ of the $d$ components of $\mathrm{y}=\psi\left(\theta_{M, 0}\right)$ followed by the $M$ blocks of $d$ components $\Psi_{M, i}=\psi\left(\theta_{M, i}\right), i=1, \ldots, M$. This notation emphasizes the special role of the first $d$ components corresponding to $\theta_{M, 0}=0$ 
which are involved when imposing the domain condition. Let us introduce the reconstruction operator $\mathcal{R}_{M}: Y_{M} \rightarrow Y$ associating to any $\left(\mathrm{y}, \Psi_{M}\right) \in Y_{M}$ the unique $M$-degree $\mathbb{R}^{d}$-valued polynomial in $Y$ interpolating y at $\theta_{M, 0}$ and $\Psi_{M, i}$ at the nodes $\theta_{M, i}, i=1, \ldots, M$. Finally, as last ingredient, we introduce the function $G_{M}: Y_{M} \rightarrow \mathbb{R}^{d}$ given by

$$
G_{M}\left(\left(\mathrm{y}, \Psi_{M}\right)\right)=G\left(\mathcal{R}_{M}\left(\mathrm{y}, \Psi_{M}\right)\right),\left(\mathrm{y}, \Psi_{M}\right) \in Y_{M},
$$

which inherits the smoothness properties of $G$. When the evaluation of $G$ requires suitable approximation techniques, we will use the notation $\widetilde{G}, \widetilde{G}_{M}$. This may happen, for instance, when $G$ involves integrals, which may need to be approximated by quadrature formulas. Hereafter, in order not to overload the notation, we avoid to use brackets when elements of $Y_{M}$ appear as input to functionals or operators. Hence, for instance, we write $G_{M}\left(\mathrm{y}, \Psi_{M}\right)$ instead of $G_{M}\left(\left(\mathrm{y}, \Psi_{M}\right)\right)$.

The discretization of $\mathcal{A}_{G}$ of index $M$ is the operator $\mathcal{A}_{G, M}: Y_{M} \rightarrow Y_{M}$ which associates to any $\left(\mathrm{y}, \Psi_{M}\right) \in Y_{M}$ the vector

$$
\mathcal{A}_{G, M}\left(\mathrm{y}, \Psi_{M}\right):=\left(v, \Upsilon_{M}\right) \in Y_{M},
$$

where

$$
\begin{gathered}
v=G_{M}\left(\mathrm{y}, \Psi_{M}\right), \\
\Upsilon_{M, i}=\left.\frac{d}{d \theta} \mathcal{R}_{M}\left(\mathrm{y}, \Psi_{M}\right)(\theta)\right|_{\theta=\theta_{M, i}}, i=1, \ldots, M .
\end{gathered}
$$

Note that (2.13) and (2.14) are the discrete version of, respectively, the domain condition in (2.5) and of the action (2.4) of $\mathcal{A}_{G}$. As for $\mathcal{A}_{G}$, the nonlinearity of $G$ affects (2.13) only. Accordingly, for the linear DDE (2.6), $\mathcal{A}_{L, M}$ is a linear finite-dimensional operator.

We have now arrived at the core of the approach. By means of the discrete operator $\mathcal{A}_{G, M}$ we can turn the ADE (2.7) into the ODE on $Y_{M}$

$$
\frac{d}{d t}\left(y_{M}(t), V_{M}(t)\right)=\mathcal{A}_{G, M}\left(y_{M}(t), V_{M}(t)\right), t \geq 0,
$$

and the abstract Cauchy problem (2.8) into the initial value problem

$$
\left\{\begin{array}{l}
\frac{d}{d t}\left(y_{M}(t), V_{M}(t)\right)=\mathcal{A}_{G, M}\left(y_{M}(t), V_{M}(t)\right), t \geq 0 \\
\left(y_{M}(0), V_{M}(0)\right)=\left(\mathrm{y}, \Psi_{M}\right)
\end{array}\right.
$$

where $\mathrm{y}=\psi(0)$ and $\Psi_{M, i}=\psi\left(\theta_{M, i}\right), i=1, \ldots, M$.

To find a representation of the discrete operator $\mathcal{A}_{G, M}$, we express $\mathcal{R}_{M}\left(\mathrm{y}, \Psi_{M}\right) \in Y$ in the Lagrange form

$$
\mathcal{R}_{M}\left(\mathrm{y}, \Psi_{M}\right)(\theta)=\ell_{M, 0}(\theta) \mathrm{y}+\sum_{i=1}^{M} \ell_{M, i}(\theta) \Psi_{M, i}, \quad \theta \in[-\tau, 0],
$$

where $\ell_{M, i}, i=0,1, \ldots, M$, are the Lagrange polynomials corresponding to (2.10), i.e.,

$$
\ell_{M, i}(\theta)=\prod_{\substack{k=0 \\ k \neq i}}^{M} \frac{\theta-\theta_{M, k}}{\theta_{M, i}-\theta_{M, k}}, i=0,1, \ldots, M .
$$


Accordingly, we define the $M \times M$ matrix

$$
D_{M}:=\left(\begin{array}{ccc}
\ell_{M, 1}^{\prime}\left(\theta_{M, 1}\right) & \ldots & \ell_{M, M}^{\prime}\left(\theta_{M, 1}\right) \\
\vdots & \ddots & \vdots \\
\ell_{M, 1}^{\prime}\left(\theta_{M, M}\right) & \ldots & \ell_{M, M}^{\prime}\left(\theta_{M, M}\right)
\end{array}\right) .
$$

For the entries of $D_{M}$ corresponding to (2.11) there are explicit formulas, see, e.g., [28] or [41]. Note that for all $\theta \in[-\tau, 0]$

$$
\sum_{i=0}^{M} \ell_{M, i}(\theta)=1
$$

and hence

$$
\sum_{i=0}^{M} \ell_{M, i}^{\prime}(\theta)=0
$$

The relations $(2.16),(2.18)$ and the matrix (2.17) enable us to express (2.13) as

$$
v=G_{M}\left(\mathrm{y}, \Psi_{M}\right)=G\left(\ell_{M, 0}(\cdot) \mathrm{y}+\sum_{i=1}^{M} \ell_{M, i}(\cdot) \Psi_{M, i}\right)
$$

and (2.14) in the compact form

$$
\Upsilon_{M}=\left(D_{M} \otimes I_{d}\right)\left(\Psi_{M}-\mathrm{e} \otimes \mathrm{y}\right)=\left(D_{M} \otimes I_{d}\right) \Psi_{M}-D_{M} \mathrm{e} \otimes \mathrm{y}
$$

where e $:=(1, \ldots, 1)^{T} \in \mathbb{R}^{M}$ and $\otimes$ is the tensor product. As a consequence of (2.13) and (2.19), the ODE (2.15) reads

$$
\left\{\begin{array}{l}
y_{M}^{\prime}(t)=G_{M}\left(y_{M}(t), V_{M}(t)\right) \\
V_{M}^{\prime}(t)=\left(D_{M} \otimes I_{d}\right) V_{M}(t)-D_{M} \mathrm{e} \otimes y_{M}(t),
\end{array} \quad t \geq 0 .\right.
$$

For a given $\psi \in \operatorname{dom}\left(\mathcal{A}_{G}\right)$, the solution $\left(y_{M}(t), V_{M}(t)\right)$ of the initial value problem for $(2.20)$ with initial condition

$\mathrm{y}=\psi(0)$ and $\Psi_{M, i}=\psi\left(\theta_{M, i}\right), i=1, \ldots, M$, provides the pseudospectral approximation of the classical solution $v(t)=y_{t}$ of $(2.8)$, that is $y_{M}(t) \approx y(t), V_{M, i}(t) \approx y\left(t+\theta_{M, i}\right), i=1, \ldots, M$, and $\mathcal{R}_{M}\left(y_{M}(t), V_{M}(t)\right) \approx v(t)=y_{t}, t \geq 0$. Therefore, the pseudospectral discretization allows to construct numerical solutions of (2.2) and, within this context, it represents an alternative to the numerical method based on Runge-Kutta discretization schemes presented in [36]. From (2.20) it is clear that the matrix $D_{M}$ corresponding to (2.11) plays a crucial role in the convergence analysis of solutions. The first fundamental results on $D_{M}$ and its spectrum have been established in the context of convergence analysis of the pseudospectral method for first order hyperbolic partial differential equations [27, 28, 29]. Here we only recall the following result, which is indeed valid for any mesh of distinct points (2.10).

Proposition 2.1. For any $M$, the matrix $D_{M}(2.17)$ is non-singular. 
Proof. Assume $D_{M}$ is a singular matrix. Then there exists $W \in \mathbb{R}^{M} \backslash\{0\}$ s.t.

$$
D_{M} W=0
$$

and the polynomial $p_{M}:=\mathcal{R}_{M}(0, W)$ is nonzero. From (2.16), (2.17) and (2.21) we easily obtain that $p_{M}^{\prime}\left(\theta_{M, i}\right)=0, i=1, \ldots, M$. Therefore $p_{M}^{\prime} \equiv 0$ and $p_{M}$ is a constant function in $[-\tau, 0]$. Since $p_{M}\left(\theta_{M, 0}\right)=0$, we get that all the components of $W$ must be zero, in contradiction with $W \neq 0$.

2.2. Stability and bifurcation of equilibria. In the theory of dynamical systems, the stability and bifurcation analysis of equilibria is a central subject. Moreover, the main tool for the local stability analysis for either ODEs or DDEs, REs and coupled REs/DDEs is the principle of linearized stability. With the aim to tackle the problem for the nonlinear DDE (2.1) by means of the nonlinear ODE (2.15), we first characterize the equilibria of (2.1) and (2.15) and next their relationship, and subsequently we study the linearization. Finally, the result on the accuracy of approximation of the eigenvalues of $\mathcal{A}_{L}$ by the eigenvalues of $\mathcal{A}_{L, M}$ given in $[6,9]$ allows us to confirm the reliability of the pseudospectral discretization for the stability and bifurcation analysis of equilibria of parametrized DDEs.

Definition 2.2. An equilibrium for (2.1) is a constant mapping $\bar{y} \in Y$ with value $\bar{y}$ s.t. $G(\bar{y})=0$. Note that we use the same symbol $\bar{y}$ to denote either the constant function, which is an element of $Y$, or its value, which is an element of $\mathbb{R}^{d}$. The meaning will be clear from the context.

For the linear DDE (2.6), the stability of the zero equilibrium is determined by the spectrum $\sigma\left(\mathcal{A}_{L}\right)$ of the associated infinitesimal generator $\mathcal{A}_{L}$, which is pure point spectrum. It is known that the zero solution is exponentially stable if and only if $\Re(\lambda)<0$ for all $\lambda \in \sigma\left(\mathcal{A}_{L}\right)$ and it is unstable if there is at least one eigenvalue $\lambda \in \sigma\left(\mathcal{A}_{L}\right)$ with positive real part.

According to the principle of linearized stability, one can turn the stability analysis of the equilibria of the nonlinear DDE (2.1) into the stability analysis of the zero solution of the linearized equation

$$
y^{\prime}(t)=D G(\bar{y}) y_{t}, t \geq 0,
$$

where $D G(\bar{y})$ denotes the Fréchet derivative of $G$ at $\bar{y}$. In fact, $\bar{y}$ is locally exponentially stable if the zero solution of (2.22) is exponentially stable, and $\bar{y}$ is unstable if the zero solution of (2.22) is unstable [18].

Now we consider the equilibria of the ODE (2.15).

Definition 2.3. An equilibrium for (2.15) is a constant solution $\left(\bar{y}, \bar{V}_{M}\right) \in Y_{M}$ s.t.

$$
\mathcal{A}_{G, M}\left(\bar{y}, \bar{V}_{M}\right)=0,
$$

that is

$$
\begin{aligned}
G_{M}\left(\bar{y}, \bar{V}_{M}\right) & =0, \\
\left.\frac{d}{d \theta} \mathcal{R}_{M}\left(\bar{y}, \bar{V}_{M}\right)(\theta)\right|_{\theta=\theta_{M, i}} & =0, \quad i=1, \ldots, M .
\end{aligned}
$$


Theorem 2.4. If the constant mapping $\bar{y} \in Y$ is an equilibrium for (2.1), then $\left(\bar{y}, \bar{V}_{M}\right)$ with

$$
\bar{V}_{M, i}=\bar{y}, i=1, \ldots, M
$$

is an equilibrium for (2.15). Vice-versa, if $\left(\bar{y}, \bar{V}_{M}\right)$ is an equilibrium for (2.15), then (2.24) holds and $\bar{y}$ is an equilibrium for (2.1).

Proof. Let $\bar{y}$ be an equilibrium of (2.1) and define the vector $\bar{V}_{M}$ as in (2.24). Since $p_{M}:=\mathcal{R}_{M}\left(\bar{y}, \bar{V}_{M}\right)$ is the constant function of value $\bar{y}$, we have that $G_{M}\left(\bar{y}, \bar{V}_{M}\right)=G(\bar{y})=0$ and, moreover, $p_{M}^{\prime}\left(\theta_{M, i}\right)=0, i=1, \ldots, M$. Then $\left(\bar{y}, \bar{V}_{M}\right)$ is an equilibrium for (2.15). Conversely, let $\left(\bar{y}, \bar{V}_{M}\right)$ be an equilibrium for (2.15). From (2.23) we have that $\mathcal{R}_{M}\left(\bar{y}, \bar{V}_{M}\right)$ is a constant polynomial and therefore $\bar{V}_{M, i}=\bar{y}, i=1, \ldots, M$. Let $\bar{y} \in Y$ be the constant mapping with value $\bar{y}$. Since $G(\bar{y})=G_{M}\left(\bar{y}, \bar{V}_{M}\right)=0$, the assertion follows.

After having established that there is a one-to-one correspondence between the equilibria via the relation (2.24), we move on to study local stability by linearization.

Theorem 2.5 (commutativity).

Linearization and pseudospectral discretization commute.

Proof. Consider the linearized DDE (2.22). The corresponding approximating ODE is obtained by replacing the first equation in (2.15) with

$$
y_{M}^{\prime}(t)=D G(\bar{y}) \mathcal{R}_{M}\left(y_{M}(t), V_{M}(t)\right), t \geq 0 .
$$

When we linearize (2.15), by (2.24) we get exactly the same result.

Now the focus is on the linearized equations: the stability properties of the equilibrium $\bar{y}$ of (2.1) and of the corresponding equilibrium $\left(\bar{y}, \bar{V}_{M}\right)$ of (2.15) given by (2.24) are determined by the location in the complex plane of the eigenvalues, respectively, of the infinitesimal generator $\mathcal{A}_{L}$ and of the matrix $\mathcal{A}_{L, M}$, where $L=D G(\bar{y})$. The accuracy of the approximation of the eigenvalues is given in the following theorem, which is valid for (2.11).

Theorem 2.6.[6,9] Let $\lambda$ be an eigenvalue of $\mathcal{A}_{L}$ of multiplicity $m$. Then, for sufficiently large $M$, there exist $m$ eigenvalues $\lambda_{M, 1}, \ldots, \lambda_{M, m}$ of $\mathcal{A}_{L, M}$, each counted with its multiplicity, s.t.

$$
\max _{i=1, \ldots, m}\left|\lambda_{M, i}-\lambda\right| \leq C_{2}\left(\frac{1}{\sqrt{M}}\left(\epsilon+\frac{C_{1}}{M}\right)^{M}\right)^{\frac{1}{m}}
$$

where $\epsilon$ takes into account the possible error in the approximation of the operator $L$, the constants $C_{1}$ and $C_{2}$ are independent of $M$ and $C_{1}$ is proportional to $|\lambda| \tau$.

An error bound of the kind (2.25) is known as spectral accuracy [41]. Since the constant $C_{1}$ is proportional to $|\lambda|$, we can obtain very accurate approximations of the eigenvalues close to the origin with small $M$. Usually, and in practice, an order of tens is sufficient to reach the machine precision, modulo multiplicity, also for the rightmost eigenvalues. The determination of the minimum discretization index $M$ ensuring a prescribed accuracy for the rightmost roots has been considered in [42] for the DDE (2.1) without distributed delay terms. Further remarks can be found in [9].

Since bifurcation of equilibria occurs when, by changing a parameter, an eigenvalue or a conjugate pair of eigenvalues passes through the imaginary axis, by collecting all the previous 
results we can conclude that the linearized version of (2.15) gives accurate information about both the stability of equilibria of the original DDE (2.1) and the parameter values at which such equilibria undergo a bifurcation.

Corollary 2.7 (numerical bifurcation). The linearization of the ODE (2.15) allows to determine the local stability or instability as well as the parameter values corresponding to bifurcation of an equilibrium of the nonlinear DDE (2.1) with spectral accuracy.

For parametrized nonlinear ODEs, well-developed numerical techniques and software for numerical continuation and bifurcation are available. It is important to remark that the dimension of the ODE (2.15) is $d(M+1)$. We expect that the spectral accuracy allows to obtain reliable results with systems whose dimension is low enough to enable efficient performance of the numerical methods. Hence, concerning the bifurcation analysis of equilibria of (2.1), the use of such tools to study (2.15) can yield trustworthy results, as shown in $\S 5$.

After having discussed in detail the case of DDEs, we extract and summarize schematically the fundamental steps that form our recipe for the numerical bifurcation analysis of general nonlinear delay equations:

(i) the initial value problem associated with the nonlinear delay equation generates a dynamical system on an infinite-dimensional function space, where the evolution semigroup is translation with a rule for extension, and the corresponding generator is a derivative operator;

(ii) through such generator, the original delay equation is reformulated as an equivalent $\mathrm{ADE}$;

(iii) the ADE is discretized using the pseudospectral method in order to obtain the approximating nonlinear ODE via Lagrange representation;

(iv) equilibria of the delay equation are in one-to-one correspondence with equilibria of the approximating ODE;

(v) pseudospectral discretization and linearization around an equilibrium commute;

(vi) the relevant eigenvalues of the linearized infinite-dimensional generator are spectrally approximated by the eigenvalues of the linearized ODE.

In the following sections we show how this template can be applied specifically to different classes of delay models, in particular REs and coupled REs/DDEs.

3. Renewal equations. REs are functional equations expressing the value of the function itself in terms of the history. A nonlinear autonomous RE is a relation of the form

$$
x(t)=F\left(x_{t}\right), t \geq 0,
$$

where the state $x_{t}$, i.e., $x_{t}(\theta)=x(t+\theta), \theta \in[-\tau, 0]$, lives in the Banach space $X:=$ $\mathcal{L}^{1}\left([-\tau, 0] ; \mathbb{R}^{d}\right)$, equipped with the norm $\|\varphi\|_{X}=\int_{-\tau}^{0}|\varphi(\theta)| d \theta, \varphi \in X$, and $F: X \rightarrow \mathbb{R}^{d}$ is a smooth nonlinear map. The choice of $X$ is motivated by the applications to population dynamics (see [17] and the references therein).

The basic results for such equations, as well as for coupled REs/DDEs, have been developed in [17], making available all the key ingredients to extend the pseudospectral discretization also to the nonlinear RE (3.1), i.e., the well-posedness of the associated initial value problem, the abstract formulation, the principle of linearized stability and the bifurcation theorems. The last ingredient to complete the program, i.e., the theorem on the convergence of the eigenvalues of the discretized generator to the eigenvalues of the original one in the linear 
case, can be obtained from [5]. We remark that the same convergence problem is addressed in [7] for a different choice of the underlying state space, namely $X=\mathcal{C}\left([-\tau, 0] ; \mathbb{R}^{d}\right)$. In this section we introduce the necessary notation and results to arrive to the abstract reformulation of (3.1) and then we examine the pseudospectral discretization, emphasizing the differences w.r.t. DDEs.

Given $\varphi \in X$, the initial value problem for (3.1) with initial condition

$$
x(\theta)=\varphi(\theta), \theta \in[-\tau, 0],
$$

has a unique solution, which depends continuously on $\varphi$. From now on we proceed as in $\S 2$. The generator of the solution semigroup associated with (3.1) is the nonlinear operator $\mathcal{A}_{F}: \operatorname{dom}\left(\mathcal{A}_{F}\right) \subseteq X \rightarrow X$

$$
\begin{gathered}
\mathcal{A}_{F}(\varphi)=\varphi^{\prime}, \varphi \in \operatorname{dom}\left(\mathcal{A}_{F}\right), \\
\operatorname{dom}\left(\mathcal{A}_{F}\right)=\{\varphi \in X \mid \varphi \in A C, \varphi(0)=F(\varphi)\},
\end{gathered}
$$

where the notation $\varphi \in A C$ means that $\varphi$ is absolutely continuous. Note that its action is still differentiation, but now the domain condition in (3.4) involves the value of $\varphi$ at 0 .

The operator (3.3-3.4) allows to define the ADE on $X$

$$
\frac{d}{d t} u(t)=\mathcal{A}_{F}(u(t)), t \geq 0,
$$

and it can be proved that the abstract Cauchy problem

$$
\left\{\begin{array}{l}
\frac{d}{d t} u(t)=\mathcal{A}_{F}(u(t)), t \geq 0 \\
u(0)=\varphi
\end{array}\right.
$$

is equivalent to the initial value problem $(3.1-3.2)$, that is $u(t)=x_{t}$ is a (classical or mild) solution of (3.6) whenever $x$ is a solution of (3.1-3.2).

Following the approach introduced in $\S 2.1$, we now construct the discretization of (3.33.4). As we will see soon, the only difference consists in how we impose the domain condition in (3.4) to the interpolating polynomial relevant to the mesh (2.11).

For a given $M \in \mathbb{N}$, let $\Omega_{M}$ be the mesh in (2.9). Every $\varphi \in X$ is discretized by the vector $\Phi_{M} \in X_{M}:=\left(\mathbb{R}^{d}\right)^{\Omega_{M}} \cong \mathbb{R}^{d M}$, which is a column vector with $M$ blocks of $d$ components $\Phi_{M, i}=\varphi\left(\theta_{M, i}\right), i=1, \ldots, M$. For a given $\mathrm{x} \in \mathbb{R}^{d}$ and $\Phi_{M} \in X_{M}$ we consider the $M$-degree polynomial $\mathcal{R}_{M}\left(\mathrm{x}, \Phi_{M}\right)$ interpolating $\mathrm{x} \in \mathbb{R}^{d}$ at $\theta_{M, 0}=0$ and $\Phi_{M, i}$ at $\theta_{M, i}, i=1, \ldots, M$, and we define the function $F_{M}: \mathbb{R}^{d} \times X_{M} \rightarrow \mathbb{R}^{d}$ by

$$
F_{M}\left(\mathrm{x}, \Phi_{M}\right):=F\left(\mathcal{R}_{M}\left(\mathrm{x}, \Phi_{M}\right)\right), \mathrm{x} \in \mathbb{R}^{d}, \Phi_{M} \in X_{M}
$$

The discretization $\mathcal{A}_{F, M}$ of index $M$ of (3.3-3.4) is the operator $\mathcal{A}_{F, M}: X_{M} \rightarrow X_{M}$ which associates to any $\Phi_{M} \in X_{M}$ the vector

$$
\mathcal{A}_{F, M}\left(\Phi_{M}\right):=\Xi_{M} \in X_{M}
$$


where

$$
\Xi_{M, i}=\left.\frac{d}{d \theta} \mathcal{R}_{M}\left(\mathrm{x}, \Phi_{M}\right)(\theta)\right|_{\theta=\theta_{M, i}}, i=1, \ldots, M,
$$

and the value $\mathrm{x}$ in (3.7) is the solution of the nonlinear equation

$$
\mathrm{x}=F_{M}\left(\mathrm{x}, \Phi_{M}\right) .
$$

Relations (3.7) and (3.8) are the discrete version of, respectively, the action (3.3) and the domain condition (3.4). Note that, contrary to DDEs, the discretized domain condition (3.8) is involved in (3.7). In many examples of REs arising in population dynamics [20], the equation (3.8) is linear w.r.t. $\mathrm{x}$ and explicitly solvable:

$$
\mathrm{x}=H_{M}\left(\Phi_{M}\right) .
$$

In general, when the matrix $I_{d}-D_{\mathrm{x}} F_{M}\left(\mathrm{x}, \Phi_{M}\right)$ is invertible in a neighborhood of the solution, the nonlinear equation (3.8) has a local unique solution (3.9), which can be computed by efficient numerical methods [38]. Hereafter we assume that (3.8) has a unique local solution (3.9). When a solver for nonlinear equations is needed, we employ the notation $\widetilde{H}_{M}$. Similarly, $\widetilde{F}$ and $\widetilde{F}_{M}$ stress the use of approximation techniques to evaluate the function $F$ and, consequently, $F_{M}$.

By means of $\mathcal{A}_{F, M}$ we turn the $\mathrm{ADE}$ (3.5) and the associated Cauchy problem (3.6) into, respectively, the ODE

$$
U_{M}^{\prime}(t)=\mathcal{A}_{F, M}\left(U_{M}(t)\right), t \geq 0,
$$

and the initial value problem

$$
\left\{\begin{array}{l}
U_{M}^{\prime}(t)=\mathcal{A}_{F, M}\left(U_{M}(t)\right), t \geq 0 \\
U_{M}(0)=\Phi_{M}
\end{array}\right.
$$

where $\Phi_{M, i}=\varphi\left(\theta_{M, i}\right), i=1, \ldots, M$. Under the assumption (3.9), by using the Lagrange representation for the polynomial $\mathcal{R}_{M}\left(\mathrm{x}, \Phi_{M}\right)$ and the matrix $D_{M}$ in (2.17), we obtain the following compact representation for (3.7)

$$
\Xi_{M}=\left(D_{M} \otimes I_{d}\right) \Phi_{M}-D_{M} \mathrm{e} \otimes H_{M}\left(\Phi_{M}\right), t \geq 0,
$$

and, as a consequence, the ODE (3.10) reads

$$
U_{M}^{\prime}(t)=\left(D_{M} \otimes I_{d}\right) U_{M}(t)-D_{M} \mathrm{e} \otimes H_{M}\left(U_{M}(t)\right), t \geq 0 .
$$

Remark 3.1. The expression (3.9) for the solution of the equation (3.8) is essential to obtain the ODE (3.11), whose dynamics can be analyzed by the existing numerical software. Note that (3.11) can be derived by inserting (3.9) into the following differential-algebraic equation

$$
\left\{\begin{array}{l}
x_{M}(t)=F_{M}\left(x_{M}(t), U_{M}(t)\right), \\
U_{M}^{\prime}(t)=\left(D_{M} \otimes I_{d}\right) U_{M}(t)-D_{M} e \otimes x_{M}(t),
\end{array} \quad t \geq 0\right.
$$


where the discretized domain condition appears explicitly.

We now briefly analyze the relation between equilibria and stability of the delay equation (3.1) and its discretization.

Definition 3.2. An equilibrium for (3.1) is a constant mapping $\bar{x} \in X$ with value $\bar{x}$ s.t. $\bar{x}=F(\bar{x})$.

Definition 3.3. An equilibrium for (3.10) is a constant mapping $\bar{U}_{M} \in X_{M}$ s.t.

$$
\mathcal{A}_{F, M}\left(\bar{U}_{M}\right)=0
$$

that is

$$
\left.\frac{d}{d \theta} \mathcal{R}_{M}\left(H_{M}\left(\bar{U}_{M}\right), \bar{U}_{M}\right)(\theta)\right|_{\theta=\theta_{M, i}}=0, i=1, \ldots, M
$$

Like for DDEs, the correspondence of equilibria is ensured for the RE (3.1).

Theorem 3.4.If the constant mapping $\bar{x} \in X$ is an equilibrium for (3.1), then $\bar{U}_{M}$ with

$$
\bar{U}_{M, i}=\bar{x}, i=1, \ldots, M,
$$

is an equilibrium for (3.10). Vice-versa, if $\bar{U}_{M}$ is an equilibrium for (3.10), then (3.13) holds with $\bar{x}=H_{M}\left(\bar{U}_{M}\right)$ and $\bar{x}$ is an equilibrium for (3.1).

Proof. Let $\bar{x} \in X$ be an equilibrium of (3.1) and define $\bar{U}_{M}$ as in (3.13). Since $p_{M}:=$ $\mathcal{R}_{M}\left(\bar{x}, \bar{U}_{M}\right)$ is the constant polynomial with value $\bar{x}$, we have that $F_{M}\left(\bar{x}, \bar{U}_{M}\right)=F(\bar{x})=\bar{x}$, and $p_{M}^{\prime}\left(\theta_{M, i}\right)=0, i=1, \ldots, M$. From $\bar{x}=H_{M}\left(\bar{U}_{M}\right)$, we conclude that $\bar{U}_{M}$ is an equilibrium for (3.10). Conversely let $\bar{U}_{M}$ be an equilibrium for (3.10) and define $\bar{x}=H_{M}\left(\bar{U}_{M}\right)$ the solution of $\bar{x}=F_{M}\left(\bar{x}, \bar{U}_{M}\right)$. From (3.12) we have that $\mathcal{R}_{M}\left(H_{M}\left(\bar{U}_{M}\right), \bar{U}_{M}\right)$ has constant value and then $\bar{U}_{M, i}=\bar{x}$. Let $\bar{x} \in X$ be the constant mapping with value $\bar{x}$. The assertion follows from $F(\bar{x})=F_{M}\left(\bar{x}, \bar{U}_{M}\right)=\bar{x}$.

The principle of linearized stability for (3.1) says that the stability of the equilibrium $\bar{x}$ can be determined by ascertaining the stability of the zero solution of the linearized equation

$$
x(t)=D F(\bar{x}) x_{t}, t \geq 0,
$$

where $\operatorname{DF}(\bar{x})$ is the Fréchet derivative of $F$ at $\bar{x}$. In particular, $\bar{x}$ is locally asymptotically stable if the zero solution of (3.14) is exponentially stable and it is unstable if the zero solution of (3.14) is unstable.

The theorem concerning the spectral accuracy of the IG-approach in approximating the eigenvalues of the infinitesimal generator of linear REs, which is analogous to Theorem 2.6, has been proved in [5]. Then, by the same arguments as used in $\S 2.2$, we conclude the validity of the appropriate analogues of Theorem 2.5, concerning the commutativity between pseudospectral discretization and linearization, and of Corollary 2.7, ensuring that the study of the approximating ODE (3.10) gives accurate information about the stability and bifurcation of the equilibria of the nonlinear RE (3.1). 
4. Coupled REs/DDEs. As already mentioned, in applications to physiologically structured populations, systems of a RE coupled with a DDE are central. All the necessary theoretical and numerical results are valid in general also for such delay equations, see $[5,17]$ and [21] for a recent discussion about the linearization of Daphnia-like models. Therefore, we can combine the procedures in $\S 2$ and $\S 3$ to extend the pseudospectral discretization and to derive the nonlinear ODE. In this section we briefly present the basic steps, skipping all the details, which can be easily recovered.

Let $X:=\mathcal{L}^{1}\left([-\tau, 0] ; \mathbb{R}^{d_{1}}\right)$ and $Y:=\mathcal{C}\left([-\tau, 0] ; \mathbb{R}^{d_{2}}\right)$. A nonlinear coupled RE/DDE is

$$
\left\{\begin{array}{l}
x(t)=F\left(x_{t}, y_{t}\right), \\
y^{\prime}(t)=G\left(x_{t}, y_{t}\right), \quad t \geq 0
\end{array}\right.
$$

where $x_{t} \in X, y_{t} \in Y$, and $F: X \times Y \rightarrow \mathbb{R}^{d_{1}}$ and $G: X \times Y \rightarrow \mathbb{R}^{d_{2}}$ are smooth nonlinear maps. As state space for (4.1) we consider $X \times Y$, interpreting the function $(\varphi, \psi)(\theta), \theta \in[-\tau, 0]$, as the column vector with the $d_{1}$ components of $\varphi(\theta)$ followed by the $d_{2}$ components of $\psi(\theta)$. Similarly to the previous sections, the initial value problem associated with (4.1) defines a dynamical system on $X \times Y$, such that the family of evolution operators is generated by the operator $\mathcal{A}_{F G}: \operatorname{dom}\left(\mathcal{A}_{F G}\right) \subseteq X \times Y \rightarrow X \times Y$ given by

$$
\begin{gathered}
\mathcal{A}_{F G}((\varphi, \psi))=\left(\varphi^{\prime}, \psi^{\prime}\right),(\varphi, \psi) \in \operatorname{dom}\left(\mathcal{A}_{F G}\right), \\
\operatorname{dom}\left(\mathcal{A}_{F G}\right)=\left\{\begin{array}{l|l}
(\varphi, \psi) \in X \times Y & \begin{array}{c}
\varphi \in A C, \psi \in \mathcal{C}^{1}\left([-\tau, 0] ; \mathbb{R}^{d_{2}}\right), \\
\left(\varphi, \psi^{\prime}\right)(0)=(F(\varphi, \psi), G(\varphi, \psi))
\end{array}
\end{array}\right\} .
\end{gathered}
$$

By means of $\mathcal{A}_{F G}$ it is possible to define the $\mathrm{ADE}$ and the abstract Cauchy problem equivalent to the initial value problem associated with (4.1).

For the delay equation (4.1), the domain condition in (4.3) combines the value of $\varphi \in X$ at 0 with the value of the derivative of $\psi \in Y$ at 0 and, as a consequence, its discretization uses both the approaches presented in $\S 2.1$ and $\S 3$.

Given $M \in \mathbb{N}$, we define $X_{M}$ and $Y_{M}$ as in $\S 3$ and $\S 2.1$, respectively, with the proper choice of the space dimensions $d_{1}$ and $d_{2}$. Note that in general the discretization indices of $X_{M}$ and $Y_{M}$ can be different. Any $(\varphi, \psi) \in X \times Y$ is discretized by the vector $\left(\Phi_{M},\left(\mathrm{y}, \Psi_{M}\right)\right) \in$ $X_{M} \times Y_{M}$. For a given $\mathrm{x} \in \mathbb{R}^{d_{1}}$ we introduce the functions $F_{M}: \mathbb{R}^{d_{1}} \times X_{M} \times Y_{M} \rightarrow \mathbb{R}^{d_{1}}$ and $G_{M}: \mathbb{R}^{d_{1}} \times X_{M} \times Y_{M} \rightarrow \mathbb{R}^{d_{2}}$ by

$$
\begin{aligned}
& F_{M}\left(\mathrm{x}, \Phi_{M}, \mathrm{y}, \Psi_{M}\right)=F\left(\mathcal{R}_{M}\left(\mathrm{x}, \Phi_{M}\right), \mathcal{R}_{M}\left(\mathrm{y}, \Psi_{M}\right)\right), \\
& G_{M}\left(\mathrm{x}, \Phi_{M}, \mathrm{y}, \Psi_{M}\right)=G\left(\mathcal{R}_{M}\left(\mathrm{x}, \Phi_{M}\right), \mathcal{R}_{M}\left(\mathrm{y}, \Psi_{M}\right)\right) .
\end{aligned}
$$

The discretization $\mathcal{A}_{F G, M}$ of (4.2-4.3) is obtained by combining the discretization techniques introduced for DDEs and REs, and the resulting approximating ODE on $X_{M} \times Y_{M}$ reads

$$
\left\{\begin{array}{l}
U_{M}^{\prime}(t)=\left(D_{M} \otimes I_{d_{1}}\right) U_{M}(t)-D_{M} \mathrm{e} \otimes K_{M}\left(U_{M}(t), y_{M}(t), V_{M}(t)\right), \\
y_{M}^{\prime}(t)=G_{M}\left(K_{M}\left(U_{M}(t), y_{M}(t), V_{M}(t)\right), U_{M}(t), y_{M}(t), V_{M}(t)\right), \quad t \geq 0, \\
V_{M}^{\prime}(t)=\left(D_{M} \otimes I_{d_{2}}\right) V_{M}(t)-D_{M} \mathrm{e} \otimes y_{M}(t),
\end{array}\right.
$$

where we have assumed that the solution of the nonlinear equation $\mathrm{x}=F_{M}\left(\mathrm{x}, \Phi_{M}, \mathrm{y}, \Psi_{M}\right)$ is locally expressed as

$$
\mathrm{x}=K_{M}\left(\Phi_{M}, \mathrm{y}, \Psi_{M}\right)
$$


The theorem about one-to-one correspondence of equilibria and the analogues of Theorem 2.5, Theorem 2.6 and Corollary 2.7 hold also for coupled REs/DDEs, ensuring that the study of the approximating ODE (4.4) gives accurate information about the stability and bifurcation of the equilibria of the nonlinear equation (4.1). The details and proofs can be easily recovered by combining properly the techniques of $\S 2.2$ and $\S 3$.

5. Numerical bifurcation analysis. In this section, in order to test the efficiency and flexibility of the proposed method, we complement the analysis with some numerical examples belonging to each class of nonlinear delay models analyzed so far, namely DDEs, REs and coupled REs/DDEs. The models are discretized using the technique introduced in the previous sections and the dynamical and bifurcation properties of the original equation w.r.t. some parameters are analyzed through the corresponding approximating ODE. As pointed out in the Introduction, several software products are nowadays available for the numerical analysis of ODEs. The simulations in this section are obtained using MATCont [16, 37], a continuation and bifurcation toolbox that is compatible with the widely used computing environment matlab. Among other functionalities, MatCont allows the continuation of equilibria and limit cycles, the detection and continuation of several codimension 1 and 2 bifurcations of equilibria and limit cycles and the computation of stability and normal forms.

For each example, we show the results of the bifurcation analysis of the approximating equation and we study the behavior of the absolute errors in the approximation of the bifurcation points w.r.t. $M$. Experimentally we verify the spectral accuracy guaranteed by the results in $\S 2.2$ for DDEs, and the analogous results for REs and coupled REs/DDEs.

5.1. A scalar DDE: delayed logistic equation. Our first example is the well-known delayed logistic equation

$$
y^{\prime}(t)=r y(t)[1-y(t-1)], t \geq 0 .
$$

The variables are scaled s.t. the delay and the carrying capacity are both equal to 1. Equation (5.1) was first introduced in [31]. For all values of $r$ the equation has two equilibria, $\bar{y}_{0}=0$ and $\bar{y}_{1}=1$. The trivial equilibrium is unstable for all $r>0$ and the positive equilibrium is asymptotically stable if $r \in\left(0, \frac{\pi}{2}\right)$. At $r^{*}=\frac{\pi}{2}$ a Hopf bifurcation occurs [1] and for $r>\frac{\pi}{2}$ the equation has a stable periodic solution.

Using the notation of $\S 2.1$, the function (2.12) is defined by

$$
G_{M}\left(\mathrm{y}, \Psi_{M}\right)=r \mathrm{y}\left(1-\Psi_{M, M}\right)
$$

and the approximating ODE (2.15) reads

$$
\left\{\begin{array}{l}
y_{M}^{\prime}(t)=r y_{M}(t)\left(1-V_{M, M}(t)\right), \quad t \geq 0 . \\
V_{M}^{\prime}(t)=D_{M}\left(V_{M}(t)-y_{M}(t) \mathbf{e}\right),
\end{array}\right.
$$

Figure 5.1(a) shows the output of the numerical bifurcation analysis of equation (5.2) obtained using MatCont with $M=10$ and tolerance option TOL $=10^{-10}$. By applying MatCont with varying $M$, it is possible to test the convergence of the computed Hopf bifurcation values $r_{M}^{*}$ to the exact point $r^{*}$. The numerical results in Figure 5.1(b) show that the absolute error $e_{M}:=\left|r_{M}^{*}-r^{*}\right|$ decays spectrally w.r.t. $M$ until the fixed tolerance TOL is reached, as expected from the results in $\S 2.2$. 


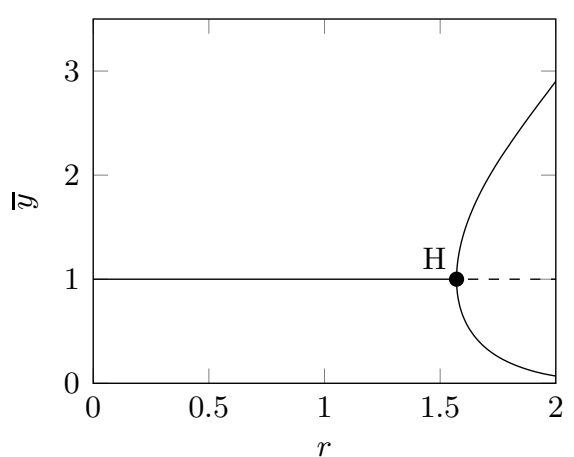

(a)

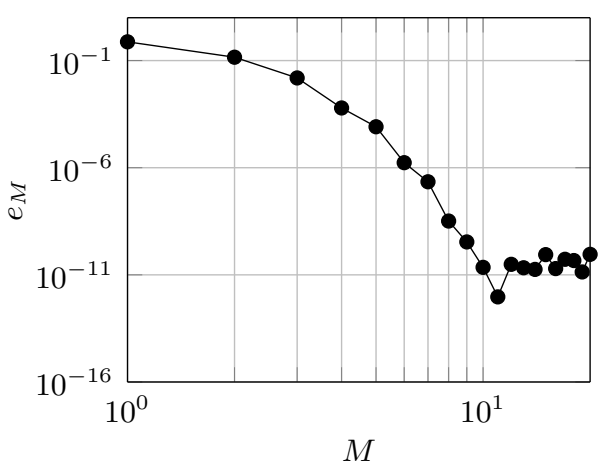

(b)

Figure 5.1. Delayed logistic model (5.1): (a) bifurcation diagram of (5.2) for $M=10$ and $T O L=10^{-10}$; (b) absolute error $e_{M}=\left|r_{M}^{*}-r^{*}\right|$ in the detected Hopf point vs. $M$.

5.2. A two dimensional DDE: predator-prey model. We consider now a DDE modeling the dynamics of a predator-prey ecological system, where the predator is structured in two classes of individuals: juveniles and adults. Only adult predators are actively hunting. We assume that, in the absence of predation, prey growth is logistic with carrying capacity $K$ and initial growth rate $r$. Predation follows mass action with coefficient $p$ and prey eaten is converted in predator offspring with a conversion coefficient $b$. At age $\tau$, juveniles become adults and start hunting. The juveniles and adults per capita mortality rates are denoted $\delta_{j}$ and $\delta_{a}$, respectively. If we denote $y_{1}(t)$ the prey density and $y_{2}(t)$ the adult predator density at time $t$, the model is described by the two dimensional DDE with a single discrete delay

$$
\left\{\begin{array}{l}
y_{1}^{\prime}(t)=r y_{1}(t)\left[1-\frac{y_{1}(t)}{K}\right]-p y_{1}(t) y_{2}(t), \\
y_{2}^{\prime}(t)=b p y_{1}(t-\tau) y_{2}(t-\tau) e^{-\delta_{j} \tau}-\delta_{a} y_{2}(t),
\end{array} \quad t \geq 0 .\right.
$$

The equilibria are $(0,0),(K, 0)$ and $\left(\bar{y}_{1}, \bar{y}_{2}\right)$, which is strictly positive if and only if

$$
p>\frac{\delta_{a}}{K b e^{-\delta_{j} \tau}} .
$$

In [26] the author conjectures that a Hopf bifurcation of the positive equilibrium occurs when the delay $\tau$ is increased.

Figure 5.2(a) represents the existence and stability regions of the nontrivial equilibrium of the discretization of (5.3) in the parameter plane $\left(\tau, \delta_{j}\right)$, approximated with MATCONT for $M=10$ and TOL $=10^{-10}$. Figure $5.2(\mathrm{~b})$ shows the approximated bifurcation diagram of the equilibrium $\left(\bar{y}_{1}, \bar{y}_{2}\right)$ w.r.t. $\tau$, obtained by numerical continuation and by analyzing the approximated eigenvalues at singularity points. Two Hopf bifurcation points are detected on the nontrivial equilibrium branch at $\tau_{1} \approx 0.2893$ and $\tau_{2} \approx 1.9416$. When positive, the equilibrium $\left(\bar{y}_{1}, \bar{y}_{2}\right)$ is unstable if $\tau \in\left(\tau_{1}, \tau_{2}\right)$, locally asymptotically stable if $\tau\left\langle\tau_{1}\right.$ or $\tau>\tau_{2}$.

In Figure 5.2(c) we can observe the convergence rate of the approximated singularity points to the reference values, which are computed with $M=20$. The branching point $\tau_{0} \approx 4.0235$, corresponding to the intersection between the trivial and nontrivial equilibrium branches, is approximated below the required tolerance TOL already for $M=1$, while the Hopf bifurcation 


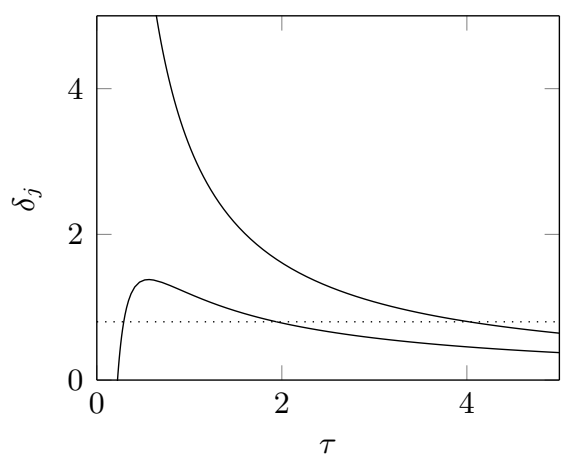

(a)

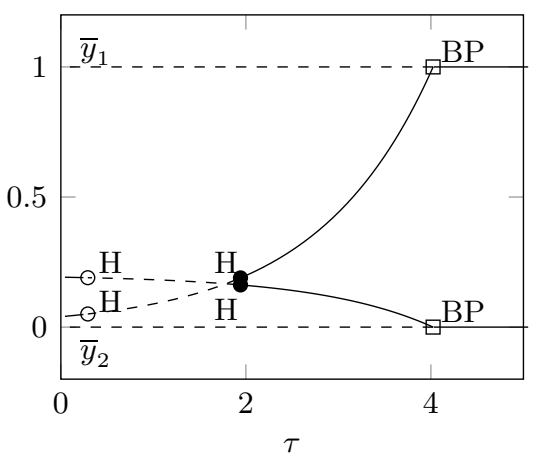

(b)

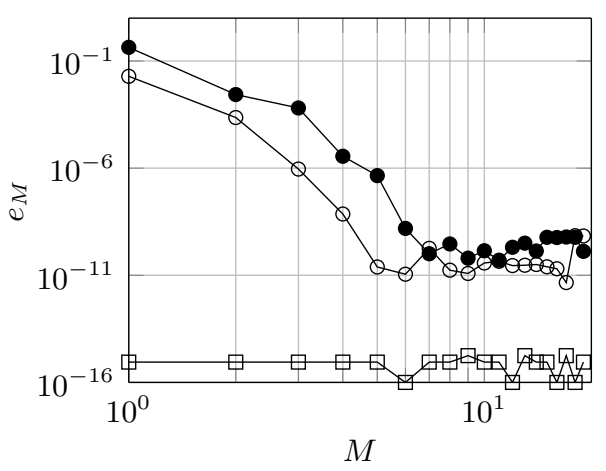

(c)

Figure 5.2. Predator-prey model (5.3): (a) approximated existence (below upper line) and stability (above lower line) regions of $\left(\bar{y}_{1}, \bar{y}_{2}\right)$ and dotted line $\delta_{j}=0.8$ in the plane $\left(\tau, \delta_{j}\right)$, for $M=10, \delta_{a}=0.2, r=K=b=1$, $p=5$ and $T O L=10^{-10}$; (b) approximated bifurcation diagram of $\left(\bar{y}_{1}, \bar{y}_{2}\right)$ w.r.t. $\tau$, for fixed $\delta_{j}=0.8$; (c) absolute error in the detected transcritical $\left(\tau_{0} \approx 4.0235, \square\right)$ and Hopf $\left(\tau_{1} \approx 0.2893, \circ\right.$, and $\left.\tau_{2} \approx 1.9416, \bullet\right)$ bifurcation points vs. $M$.

points $\tau_{1}$ and $\tau_{2}$ show spectral convergence until the tolerance TOL is reached. It is interesting to notice that the immediate convergence of the approximated branching point to the required tolerance is consistent with the result in Theorem 2.6, since the rightmost eigenvalue is $\lambda=0$ at the branching point. Similarly, the convergence trends in the Hopf points reflect the dependence on $\tau$ of the constant $C_{1}$ in Theorem 2.6: smaller delay $\tau_{1}$ requires a lower index $M$ to reach a given tolerance.

5.3. A scalar RE: cannibalism model. We now consider a caricatural egg cannibalism model. The population is structured in two classes of individuals, juveniles and adults. Individuals become adult when they reach the constant maturation age $\bar{a}>0$, they have a constant survival probability until age $a$ for all $\bar{a} \leq a \leq a_{\max }$, and they die when they reach the maximum lifespan $a_{\max }$. With some probability, newborns are eaten by adults. After scaling, the model is described by the equation

$$
x(t)=\beta \int_{\bar{a}}^{a_{\max }} x(t-a) e^{-x(t-a)} d a, t \geq 0,
$$




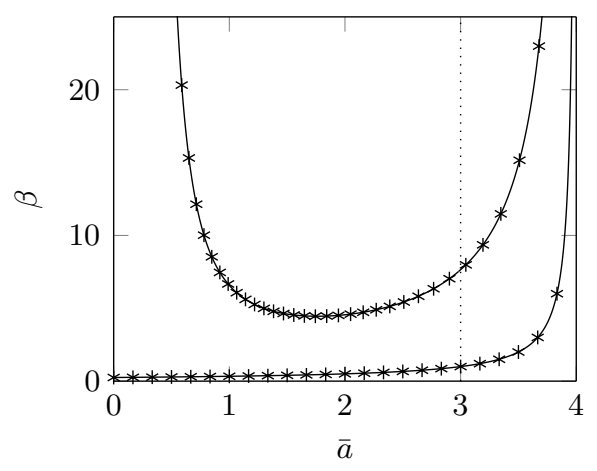

(a)

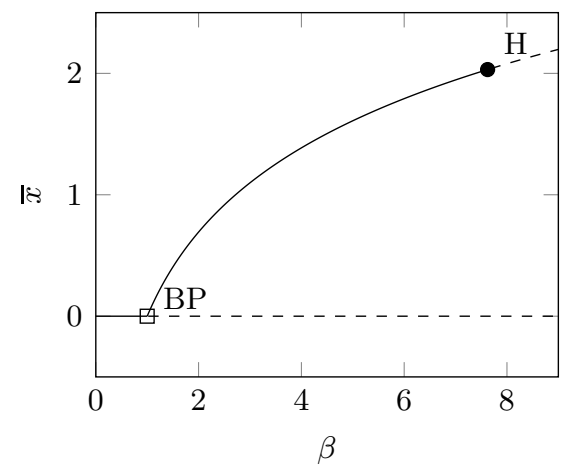

(b)

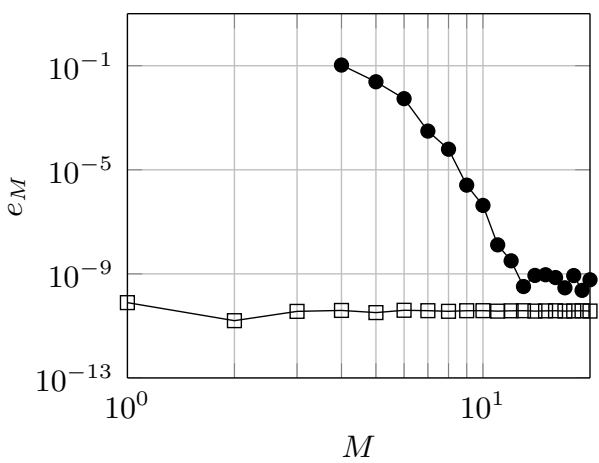

(c)

Figure 5.3. Cannibalism model (5.4): (a) approximated existence (above lower line) and stability (below upper line) regions of $\bar{x}$ in the plane $(\bar{a}, \beta)$, dotted line $\bar{a}=3$, and points on the analytical curves from [4] (*), for $M=10, a_{\max }=4$ and $T O L=10^{-10}$; (b) bifurcation diagram of (5.5) w.r.t. $\beta$, for fixed $\bar{a}=3$; (c) absolute error in the detected transcritical $\left(\beta_{B P}=1, \square\right)$ and $\operatorname{Hopf}\left(\beta_{H} \approx 7.6474, \bullet\right)$ bifurcation point of (5.5) vs. M.

where $x(t)$ denotes the adult population density at time $t$ and $\beta$ is the effective fecundity rate, i.e., the per capita number of offspring per unit of time that survive until the maturation age $\bar{a}$. The exponential term inside the integral describes the probability of newborns to survive the cannibalistic activity of adults. Equation (5.4) is a nonlinear $\mathrm{RE} \mathrm{(3.1)} \mathrm{where} d=1, \tau=a_{\max }$ and $F(\varphi)=\int_{-a_{\max }}^{-\bar{a}} \varphi(\theta) e^{-\varphi(\theta)} d \theta$. We refer to [4] for a more detailed derivation.

The trivial solution is always an equilibrium of equation (5.4). The nontrivial equilibrium $\bar{x}=\log \left[\beta\left(a_{\max }-\bar{a}\right)\right]$ is positive if $\beta\left(a_{\max }-\bar{a}\right)>1$ and at $\beta_{B P}=\left(a_{\max }-\bar{a}\right)^{-1}$ a transcritical bifurcation occurs. When the maturation age $\bar{a}$ is fixed, the positive equilibrium undergoes a Hopf bifurcation for some value $\beta_{H}$. The stability and bifurcation properties of this model have been analyzed in [4] theoretically and numerically by linearizing the equation at $\bar{x}$ and by approximating the eigenvalues of the linearized infinitesimal generator by the IG-approach for some values of the parameters $\beta$ and $\bar{a}$. Here, we perform the bifurcation analysis varying the parameter $\beta$ for the nonlinear equation (5.4) avoiding the linearization. As pointed out in $\S 3$, for a given discretization index $M$ we need to treat the domain condition (3.8), where 
the function $F_{M}$ is now defined by

$$
F_{M}\left(\mathrm{x}, \Phi_{M}\right)=\beta \int_{-a_{\max }}^{-\bar{a}} p_{M}(\theta) e^{-p_{M}(\theta)} d \theta,
$$

where $p_{M}:=\mathcal{R}_{M}\left(\mathrm{x}, \Phi_{M}\right)$. By computing numerically for all $t \geq 0$ the solution $x_{M}(t):=$ $\widetilde{H}_{M}\left(U_{M}(t)\right)$ of the nonlinear equation

$$
x_{M}(t)-F_{M}\left(x_{M}(t), U_{M}(t)\right)=0,
$$

we get the approximating ODE

$$
U_{M}^{\prime}(t)=D_{M}\left(U_{M}(t)-\widetilde{H}_{M}\left(U_{M}(t)\right) \text { e) }, t \geq 0 .\right.
$$

Figure 5.3(a) shows the existence and stability boundaries of the nontrivial equilibrium in the plane $(\bar{a}, \beta)$ approximated with MATConT, for $M=10$ and TOL $=10^{-10}$, compared to the analytical curves determined in [4]. The dotted line is the line along which the bifurcation diagram in Figure 5.3(b) is computed. In Figure 5.3(c) the absolute error between the approximated bifurcation points (branching and Hopf) and the reference values $\beta_{B P}=1$ and $\beta_{H} \approx 7.6474$, computed with $M=20$, is plotted versus the discretization index $M$, with TOL $=10^{-10}$. As already observed in the predator-prey model, the transcritical bifurcation point is approximated to the required tolerance already for $M=1$, while the Hopf bifurcation point, detected for $M \geq 4$, converges spectrally to the reference value.

5.4. A coupled RE/DDE: logistic Daphnia model. The next example is a logistic Daphnia model studied in [4]. We consider an age structured consumer population characterized by its birth rate $b(t)$ and an unstructured resource with density $S(t)$.

The dynamics is described by the following coupled RE/DDE

$$
\left\{\begin{array}{l}
b(t)=\beta S(t) \int_{\bar{a}}^{a_{\max }} b(t-a) d a, \\
S^{\prime}(t)=r S(t)\left(1-\frac{S(t)}{K}\right)-\gamma S(t) \int_{\bar{a}}^{a_{\max }} b(t-a) d a,
\end{array} \quad t \geq 0,\right.
$$

where $r$ and $K$ denote, respectively, the growth rate at small density and the carrying capacity of the resource in the absence of consumer; $\beta$ and $\gamma$ are the effective fecundity and consumption rate of the consumer until the fixed maturation age $\bar{a}$. As in the cannibalism model, we assume that adults have a constant survival probability until the maximal age $a_{\max }$.

The equation admits the equilibria $\bar{E}_{0}=(0,0), \bar{E}_{1}=(0, K)$ and the nontrivial equilibrium $\bar{E}_{2}=(\bar{b}, \bar{S})$, which is strictly positive if and only if $\beta \geq\left[K\left(a_{\max }-\bar{a}\right)\right]^{-1}$.

In [4], a Hopf bifurcation is numerically detected by linearizing the equation around $\bar{E}_{2}$ and by approximating the eigenvalues using the IG-approach. Here, we analyze the nonlinear ODE (4.4) with

$$
\begin{aligned}
F_{M}\left(\mathrm{x}, \Phi_{M}, \mathrm{y}, \Psi_{M}\right) & =\beta \mathrm{y} \int_{-a_{\max }}^{-\bar{a}} p_{M}(\theta) d \theta \\
G_{M}\left(\mathrm{x}, \Phi_{M}, \mathrm{y}, \Psi_{M}\right) & =r \mathrm{y}\left(1-\frac{\mathrm{y}}{K}\right)-\gamma \mathrm{y} \int_{-a_{\max }}^{-\bar{a}} p_{M}(\theta) d \theta
\end{aligned}
$$




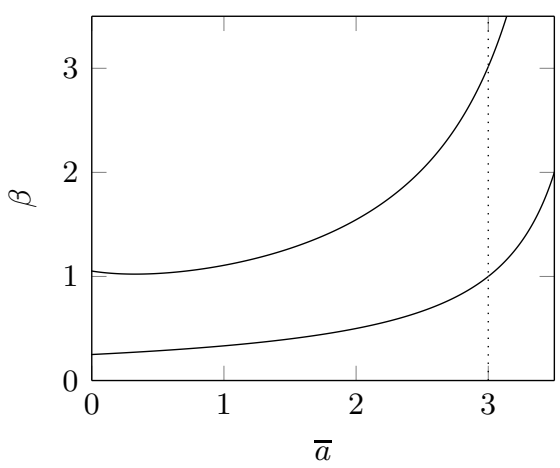

(a)

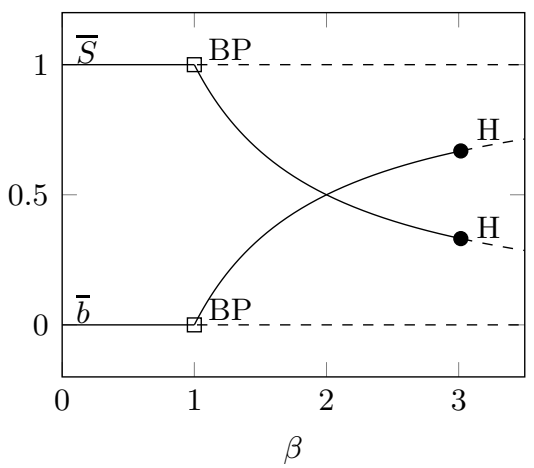

(b)

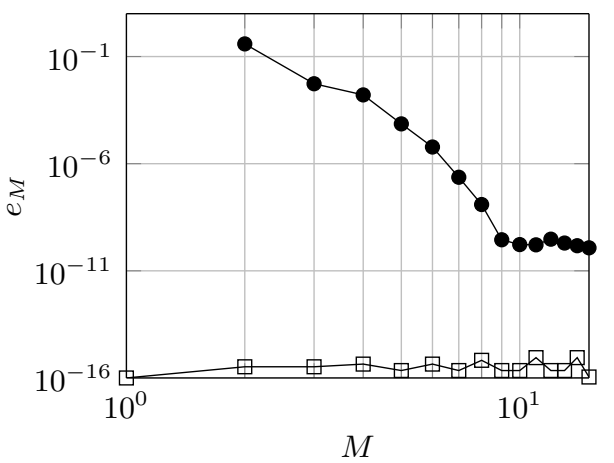

(c)

Figure 5.4. Logistic Daphnia model (5.6): (a) approximated existence (above lower line) and stability (below upper line) regions of $(\bar{b}, \bar{S})$ and dotted line $\bar{a}=3$ in the plane $(\bar{a}, \beta)$, for $M=10, r=K=\gamma=1$, $a_{\max }=4$ and $T O L=10^{-10}$; (b) approximated bifurcation diagram of $(\bar{b}, \bar{S})$ w.r.t. $\beta$, for fixed $\bar{a}=3 ;$ (c) absolute error in the detected transcritical $\left(\beta_{B P}=1, \square\right)$ and $\operatorname{Hopf}\left(\beta_{H} \approx 3.0161, \bullet\right)$ bifurcation point vs. $M$.

where $p_{M}:=\mathcal{R}_{M}\left(\mathrm{x}, \Phi_{M}\right)$. Since the function $F_{M}$ is linear w.r.t. x, we can solve it explicitly and get

$$
K_{M}\left(\Phi_{M}, \mathrm{y}, \Psi_{M}\right)=\beta \mathrm{y}\left(1-\int_{-a_{\max }}^{-\bar{a}} \ell_{0}(\theta) d \theta\right)^{-1} \sum_{i=1}^{M} \Phi_{M, i} \int_{-a_{\max }}^{-\bar{a}} \ell_{i}(\theta) d \theta .
$$

Figure 5.4(a) shows the plot of the approximated existence and stability boundary of the nontrivial equilibrium of equation (5.6) together with the reference line $\bar{a}=3$ for the bifurcation analysis, for $M=10$ and TOL $=10^{-10}$. The bifurcation diagram w.r.t. $\beta$ is plotted in Figure 5.4(b). Figure 5.4(c) shows the behavior of the absolute error in the detected branching and Hopf point w.r.t. the discretization index $M$, where TOL $=10^{-10}$. The Hopf reference value $\beta_{H} \approx 3.0161$ is computed for $M=20$. We observe that the Hopf point is detected for $M \geq 2$.

Remark 5.1. Although based on rather simplifying assumptions, the logistic Daphnia model (5.6) shows the typical features of an ecological model with age structure. Indeed, as explained in [20], physiologically structured populations are naturally described by a linear RE for the birth rate coupled with a DDE for the evolution of the environmental variable (usually, the 


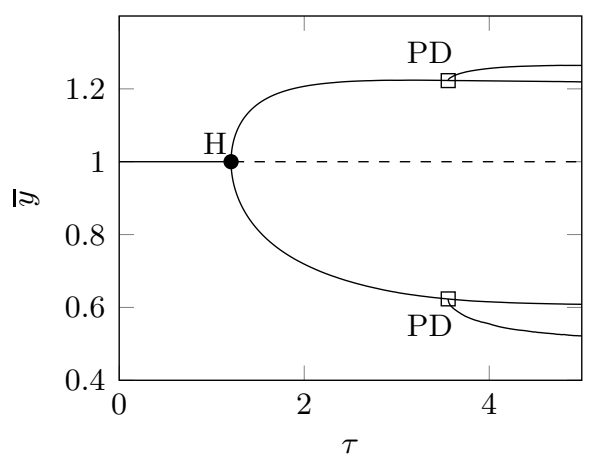

(a)

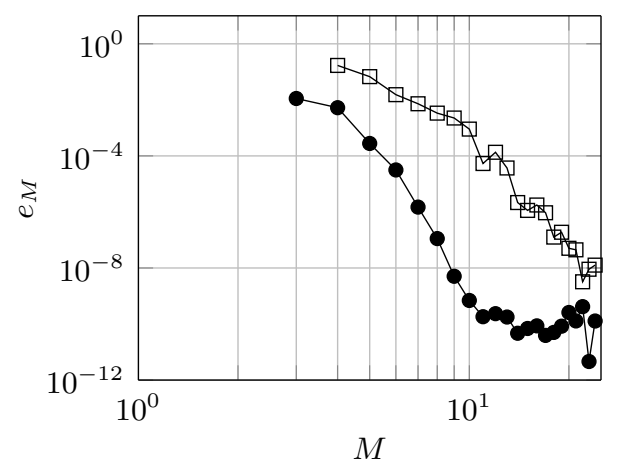

(b)

Figure 5.5. Mackey-Glass equation (5.7): (a) approximated bifurcation diagram w.r.t. $\tau$ for $M=10$, $\beta=2, \gamma=1, n=6$ and $T O L=10^{-8}$; (b) absolute error in the detected Hopf $\left(\tau_{H} \approx 1.2092, \bullet\right)$ and period doubling $\left(\tau_{P D} \approx 3.5571, \square\right)$ bifurcation point vs. $M$.

resource or substrate). The linearity w.r.t. the birth rate is an intrinsic feature of such models and it allows to obtain the explicit expression (4.5).

5.5. Bifurcation of periodic solutions: Mackey-Glass equation. We conclude this series of numerical examples with a look to future extensions. The software that is nowadays available for ODEs allow to approximate bifurcation of equilibria, periodic solutions and even more complex dynamical objects. Therefore, the pseudospectral discretization method is potentially applicable not only for the bifurcation analysis of equilibria, but also of periodic solutions. This statement requires a deeper analysis that we reserve for future research. We show here some first and promising results in this direction by applying our method to the well-known Mackey-Glass equation

$$
y^{\prime}(t)=\beta \frac{y(t-\tau)}{1+y(t-\tau)^{n}}-\gamma y(t), t \geq 0,
$$

introduced in [35] to describe the hematopoiesis process. Here, $y(t)$ denotes the density of blood cells at time $t$ and $\beta, \gamma, n$ and $\tau$ are positive parameters.

If $\beta>\gamma$, the equation has a strictly positive equilibrium $\bar{y}=\left(\frac{\beta}{\gamma}-1\right)^{1 / n}$ which is locally asymptotically stable for small values of $\tau$. For a certain value $\tau_{H}$ the nontrivial equilibrium undergoes a Hopf bifurcation and a stable periodic solution appears. Increasing the delay further, a sequence of period doubling bifurcations has been observed [35]. The results of the numerical continuation of the equilibrium branch and of the periodic solution w.r.t. $\tau$ are plotted in Figure 5.5(a) for $M=10$ and TOL $=10^{-8}$. The Hopf bifurcation is detected at $\tau_{H} \approx 1.2092$ and a period doubling bifurcation at $\tau_{P D} \approx 3.5571$. Figure 5.5(b) shows the absolute error between the detected bifurcation points and the reference values, which are computed for $M=25$. The convergence of the Hopf bifurcation point resembles the previous examples. The period doubling bifurcation shows a similar trend, although it requires larger values of $M$.

6. Conclusions and outlook. The proposed pseudospectral discretization of nonlinear delay equations appears to be an effective and flexible tool for the stability and bifurcation 
analysis of equilibria. It allows to treat not only DDEs with both discrete and distributed delays, but also REs and coupled REs/DDEs. In all cases the starting point is given by the reformulation of the nonlinear delay equation as an $\mathrm{ADE}$, which is described by a derivative operator subject to a domain condition depending on the nature and on the right-hand side of the original delay equation. The pseudospectral discretization turns the infinite-dimensional operator into a finite-dimensional one. The resulting nonlinear ODE can be easily derived and then analyzed by various efficient software packages, opening new prospects for the bifurcation analysis of delay equations. We are planning to develop further numerical tests. Moreover, for DDEs, an accurate performance comparison with DDE-BIFTOOL is in the pipeline.

The toolboxes for ODEs also support the study of more complex bifurcations. As a follow up to $\S 5.5$, we are planning to extend the analysis to bifurcation of periodic orbits, with due attention to several interesting examples. The convergence of nonconstant solutions of the initial value problem for the approximating ODE is strictly connected with the spectral properties of the matrix $D_{M}(2.17)$, and we are investigating this link in detail. Moreover, the resulting ODE could allow to approximate smooth solutions of the initial value problem associated with the delay equation. This idea has already been explored for DDEs in [36], where the discretization is based on Runge-Kutta methods. The pseudospectral discretization provides an alternative.

Among the future issues, we include also the bifurcation analysis of realistic models of physiologically structured populations of Daphnia type. The complexity of such models represents a considerable challenge and only limited tools are nowadays available, restricted to the analysis of equilibria of the linearized problems $[5,13]$. In such cases the power of the pseudospectral discretization might be essential, since it potentially provides a simple tool for a complete analysis.

Finally, in many cases of ecological modeling it is not possible to determine a priori bounds on the delay interval, e.g., $[3,17]$. In this light, we plan also to extend the pseudospectral discretization approach to delay models with infinite delay, motivated by the theory in [19].

\section{REFERENCES}

[1] J. Arino, L. Wang, And G. S. K. Wolkowicz, An alternative formulation for a delayed logistic equation, J. Theor. Biol., 241 (2006), pp. 109-119.

[2] AUTO, http://indy.cs.concordia.ca/auto/

[3] E. Beretta And D. Breda, Discrete or distributed delay? Effects on stability of population growth, Math. Biosci. Eng., to appear.

[4] D. Breda, O. Diekmann, S. Maset, and R. Vermiglio, A numerical approach for investigating the stability of equilibria for structured population models, J. Biol. Dyn., 7(1) (2013), pp. 4-20.

[5] D. Breda, P. Getto, J. SÁnchez, and R. Vermiglio, Computing the eigenvalues of realistic Daphnia models by pseudospecral methods, in print on SIAM J. Sci. Comput.

[6] D. Breda, S. Maset, And R. Vermiglio, Pseudospectral differencing methods for characteristic roots of delay differential equations, SIAM J. Sci. Comput., 27 (2005), pp. 482-495.

[7] - Pseudospectral approximation of eigenvalues of derivative operators with non-local boundary conditions, Appl. Numer. Math., 56(3-4) (2006), pp. 318-331.

[8] — Approximation of eigenvalues of evolution operators for linear retarded functional differential equations, SIAM J. Num. Anal., 50(3) (2012), pp. 1456-1483.

[9] - Stability of Linear Differential Equations - A numerical approach with Matlab, Springer Briefs in Control, Automation and Robotics, Springer, New York, 2015. 
[10] J. M. Cushing, Integrodifferential Equations and Delay Models in Population Dynamics, No. 20 in Lecture Notes in Biomathematics, Springer-Verlag, Berlin, 1977.

[11] DDE-BIFTOOL, http://twr.cs.kuleuven.be/research/software/delay/ddebiftool.shtml

[12] A. M. DE Roos, A gentle introduction to models of physiologically structured populations, in StructuredPopulation Models in Marine, Terrestrial, and Freshwater Systems, S. Tuljapurkar and H. Caswell, eds., Chapman and Hall, New York, 1997, pp. 119-204.

[13] A. M. de Roos, O. Diekmann, P. Getto, and M. A. Kirkilionis, Numerical equilibrium analysis for structured consumer resource models, B. Math. Biol., 72 (2010), pp. 259-297.

[14] A. M. De Roos, J. A. J. Metz, E. Evers, And A. Leipoldt, A size-dependent predator prey interaction: who pursues whom?, J. MATH. Biol., 28 (1990), PP. 609-643.

[15] A. M. De Roos And L. Persson, Population and Community Ecology of Ontogenetic Development, No. 51 in Monographs in Population Biology, Princeton University Press, Princeton, 2013.

[16] A. Dhooge, W. Govaerts, And Y. A. Kuznetsov, Matcont: a MATLAB package for numerical bifurcation analysis of ODEs, ACM Trans. MATH. SofTw., 29 (2003), PP. 141-164.

[17] O. Diekmann, P. Getto, And M. Gyllenberg, Stability and bifurcation analysis of Volterra functional equations in the light of suns and stars, SIAM J. Math. AnAl., 39 (2007), PP. 1023-1069.

[18] O. Diekmann, S. A. van Gils, S. M. Verduyn Lunel, and H. O. Walther, Delay Equations. Functional, Complex and Nonlinear Analysis, No. 110 in Applied Mathematical Sciences, SpringerVERlag, New York, 1995.

[19] O. Diekmann And M. Gyllenberg, Equations with infinite delay: blending the abstract and the concrete, J. Differ. Equations, 252 (2012), PP. 819-851.

[20] O. Diekmann, M. Gyllenberg, J. A. J. Metz, S. Nakaoka, and A. M. De Roos, Daphnia revisited: local stability and bifurcation theory for physiologically structured population models explained by way of an example, J. MATH. Biol., 61 (2010), PP. 277-318.

[21] O. Diekmann And K. Korvasová, Linearization of solution operators for state-dependent delay equations: a simple example, Discret. Contin. Dyn. S., 36(1) (2016), PP 137-149.

[22] Dynamical Systems Software List, http://www.Dynamicalsystems.org/sw/sw/

[23] K. Engel and R. Nagel, One-Parameter Semigroups for Linear Evolution Equations, NO. 194 IN Graduate Texts in Mathematics, Springer-Verlag, New York, 1999.

[24] T. Erneux, Applied Delay Differential Equations, No. 3 in Surveys and Tutorials in the Applied Mathematical Sciences, Springer-Verlag, New York, 2009.

[25] N. J. Ford AND V. WULF, Embedding of the numerical solution of a DDE into the numerical solution of a system of ODEs, TeChnical Report, Manchester Centre for Computational Mathematics, University of Manchester, 1998.

[26] J. E. Forde, Delay Differential Equation Models in Mathematical Biology, Ph.D. Thesis, The UniverSiTy OF Michigan, 2005.

[27] D. Gottlieb, The stability of pseudospectral-Chebyshev methods, Math. CoмP., 36(153) (1981), PP. 107-118.

[28] D. Gottlieb, M. Y. Hussaini, and S. A. Orszag, Introduction: Theory and Applications of Spectral Methods, in Spectral Methods for Partial Differential Equations, R. G. Voigt, D. Gottlieb, and M. Y. Hussaini, eds., SiAM, Philadelphia, 1984.

[29] D. Gottlieb And S. Orszag, Numerical Analysis of Spectral Methods: Theory and Applications, SIAM NSF-CBMS, No. 26, Philadelphia, 1977.

[30] J. K. Hale and S. M. Verduyn Lunel, Introduction to functional differential equations, No. 99 IN AMS series, Springer-Verlag, New York, 1993.

[31] G. E. Hutchinson, Circular causal systems in ecology, Ann. N.Y. ACAd. Sci., 50(4) (1948), PP. 221246.

[32] T. Insperger and G. StépÁn, Semi-Discretization for Time-Delay Systems: Stability and Engineering Applications, No. 178 in AMS series, Springer-Verlag, New York, 2011.

[33] KNUT, HTTP://Rs1909.GITHUB.IO/KNUT/

[34] Y. A. Kuznetsov, Elements of Applied Bifurcation Theory, No. 112 in AMS SERIES, SpringerVerlag, New York, 1998.

[35] M. C. Mackey And L. Glass, Oscillation and chaos in physiological control systems, SCIENCE, 197(4300) (1977), PP. 287-289. 
[36] S. MASET, Numerical solution of retarded functional differential equations as abstract Cauchy problems, J. Comp. Appl. Math, 161 (2003), Pp. 259-282.

[37] Matcont, http://www.matcont.ugent.Be/

[38] J. M. Ortega And W. C. Rheinboldt, Iterative solution of nonlinear equations in several variables, Academic Press, Inc., San Diego, USA, 1970.

[39] PyDSTool, HTtP://www.ni.gsu.edu/ RCLewley/PyDSTool/ProjectOverview.htmL

[40] H. L. Smith, An Introduction to Delay Differential Equations with Applications to the Life Sciences, Texts in Applied Mathematics, Springer-Verlag, New York, 2011.

[41] L. N. Trefethen, Spectral Methods in MATLAB, SiAM, Philadelphia, 2000.

[42] Z. Wu AND W. Michiels, Reliably computing all characteristic roots of delay differential equations in a given right half plane using a spectral method, J. Comput. ApPL. MATH., 236(9) (2012), PP. 24992514.

[43] XPP, HTTP://WWW.MATH.PITT.EDU/ BARD/XPP/XPP.HTML 